\title{
Preparing to be the medical registrar on call: the evolution of a simulation programme
}

\author{
Authors: Catherine Cucknell, ${ }^{\mathrm{A}}$ Elinor George ${ }^{\mathrm{A}}$ and Maria Saunders ${ }^{\mathrm{A}}$
}

\section{Introduction}

According to local and national surveys ${ }^{1}$ core medical trainees (CMTs) were anxious about the transition to becoming medical registrars. Simulation is a mandatory part of the new Internal Medical Training (IMT) curriculum, ${ }^{2}$ however, no formal simulation programme previously existed for the CMTs in our trust. Our aim was to develop a structured simulation programme and determine whether this could improve the CMTs' confidence in becoming a medical registrar.

\section{Materials and methods}

Over one academic year, four monthly simulation days with 'Step up to registrar' scenarios were piloted during the CMT teaching programme in Torbay Hospital. The scenarios gave the CMTs the opportunity to act as either the medical $\mathrm{SHO}$ or registrar, while their colleagues observed via video link.

Each scenario was followed by a micro-teach that focused on human factor awareness and clinical learning points. Throughout the programme, the CMTs were taught peer-led debriefing skills and facilitated discussions were undertaken on wellbeing topics such as recognising and preventing burnout and supporting the team. There was an escalation in the intensity and difficulty of the scenarios in each subsequent training day.

\section{Results and discussion}

Feedback concerning perceived clinical and non-clinical skill confidence and experience of simulation was collected prior to the first session and after each subsequent session. Pre-course feedback allowed us to tailor the day to areas of concern for the individual CMTs.

Utilising both CMT feedback and facilitator reflections, the programme was able to evolve. Less scenarios were used during each day of teaching to reduce learner fatigue, while ensuring individual opportunities to be both CMT and medical registrar. The scenarios themselves evolved to include more human factor stressors, such as a crying baby, abusive patients and difficult discussions with relatives.

Feedback highlighted the appreciation of more realistic simulation scenarios therefore we moved to a default position of

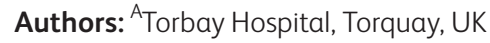

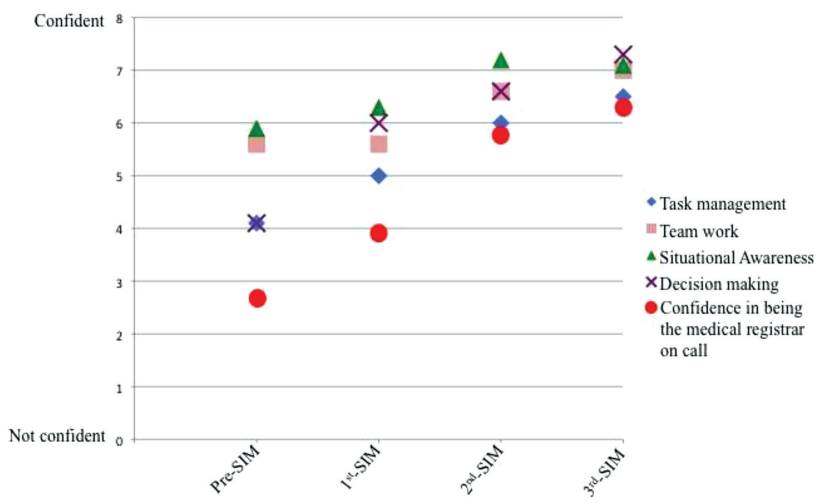

Fig 1. Participants perceived confidence in being the medical registrar and management of human factors.

using live actors or hybrid models, unless it was not possible to do so, for all scenarios eg variceal bleeding, stroke or sepsis. This created more convincing, interactive experiences. ${ }^{3}$ The overall feedback from the programme was largely positive, with the CMTs reporting a clear learning benefit (see Figs 1 and 2).

Reflecting on the feedback overall, and following each day, we were able to adapt and evolve so the CMTs got the maximum out of this learning environment. An IMT simulation programme has now been developed using the experiences and feedback from this initial pilot and the same format has been used for registrar simulation training. In order to continue evolving, we plan to

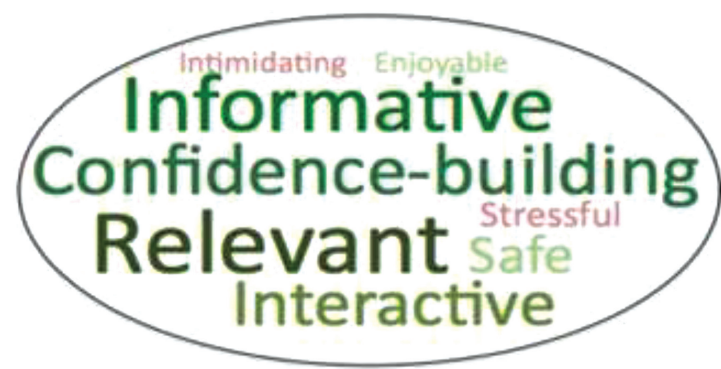

Fig 2. Word cloud descriptor feedback by frequency. 
include the use of bleeps to create the registrar on-call experience and continue to grow our scenarios to fit the learning needs of the individuals.

\section{Conclusion}

Our pilot has shown that simulation can be used to improve not only clinical confidence, but also develop human factor skills. It has also shown that by using feedback to inform continuous development, simulation can adapt to the needs of the group and can be used to train future medical registrars.

\section{Conflicts of interest}

None declared.

\section{References}

1 Tasker F, Newbery N, Burr B, Goddard A. Survey of core medical trainees in the United Kingdom 2013 - inconsistencies in training experience and competing with service demands. Clin Med 2014;14:149-56.

2 Joint Royal Colleges of Physicians Training Board. Curriculum for Internal Medicine: Stage 1 Training. London: JRCPTB, 2019:34. www.jrcptb.org.uk/sites/default/files/IM_Curriculum_Sept2519.pdf [Accessed 09 October 2019].

3 Siassakos D, Draycott T, O'Brien K, Kenyon C, Bartlett C, Fox R. Exploratory Randomized Controlled Trial of Hybrid Obstetric Simulation Training for Undergraduate Students. Simulation in Healthcare 2010;5:193-8. 